\title{
Sistema de auto-diagnóstico para evaluación de competencias de investigación en empresas tecnológicas
}

\section{Self-diagnostic system for evaluation of research skills in technology companies}

Ana Romo González Universidad Tecnológica de Jalisco Guadalajara, Jalisco - México aromo@utj.edu.mx

María de los Ángeles Villalobos A. Universidad Tecnológica de Jalisco Guadalajara, Jalisco - México avillalobos@utj.edu.mx

María de los Ángeles Márquez S. Universidad Tecnológica de Jalisco Guadalajara, Jalisco - México amarquez@utj.edu.mx

\section{Luis Manuel López Hernández \\ Universidad Tecnológica de Jalisco \\ Guadalajara, Jalisco - México llopez@utj.edu.mx}

Revista Cumbres Vol.5 №1

Versión impresa ISSN 1390-9541

Versión electrónica ISSN 1390-3365

http://investigacion.utmachala.edu.ec/revistas/index.php/Cumbres 


\title{
RESUMEN
}

Este trabajo formó parte de una investigación sobre el diseño de un Modelo de Gestión del Conocimiento (MGC) para el desarrollo de competencias de investigación del capital humano que se desempeña en empresas de alta tecnología en México. La figura primordial del MGC es el capital humano ya que al generar conocimientos incrementa la competitividad empresarial y con la producción científica y tecnológica impacta en los indicadores estandarizados internacionalmente para medir el avance económico y social del país, como la dependencia tecnológica calculada a través del registro de patentes nacionales y extranjeras. Se utilizó el método de "desarrollo de proyectos" sustentado en la definición de seis dominios de competencias que determinan el desarrollo de habilidades de investigación cuantitativamente con una escala de Likert, lo que garantiza la rápida aplicación y la obtención de resultados en línea con base en una distribución porcentual de cinco rangos. Los rangos son consistentes con las evaluaciones por competencias mediante las especificaciones de "aún no desarrollada" hasta el nivel de "Alto". Por lo que, de acuerdo con las tendencias de fomento al desarrollo y la producción científica mundiales, el presente sistema contribuye a fortalecer el área de investigación de sectores considerados estratégicos a nivel mundial.

Palabras clave: Sistema auto-diagnóstico, gestión del conocimiento, empresas tecnológicas

\begin{abstract}
This work is part of a research about the design of a Knowledge Management Model (MGC) for the development of research skills of human capital that works in high-tech companies in Mexico. In the management model, human capital is considered the primary figure of knowledge generation process that increases business competitiveness; as well as the scientific and technological production that has a direct impact on internationally standardized indices to measure economic and social progress of a country like technological dependence, calculated through the registration of domestic and foreign patents. The "project development" method was used, based on the definition of six competency domains that determine the development of research skills quantitatively with a Likert scale. that ensures rapid implementation and obtaining results on line whose final diagnosis is determined based on a percentage distribution of five ranges. The ranges are consistent with assessments by competencies through specifications like "undeveloped" to "high". So, according to the trends of promoting development and global scientific production, the present system contributes to strengthening the research of strategic sectors.
\end{abstract}

Keywords: Self-diagnosis system, research skills, high-tech enterprises

\section{Cumbres}




\section{INTRODUCCIÓN}

En México, el Plan Nacional de Desarrollo 2013-2018 (PND, 2013) pretende mediante el objetivo 4.8: "desarrollar los sectores estratégicos del país" y el Programa de Desarrollo Innovador 2013-2018 (PDI) de la Secretaría de Economía (SE, 2013) tiene como objetivo sectorial 1: "desarrollar una política de fomento industrial y de innovación que promueva un crecimiento económico equilibrado por sectores, regiones y empresas".

De acuerdo con estas tendencias, el presente desarrollo tiene como objetivo hacer un auto-diagnóstico de competencias del capital humano que contribuya al fortalecimiento del área de investigación en empresas de sectores estratégicos como Tecnologías de la Información y la Comunicación, Manufactura electrónica, manufactura automotriz y aeroespacial. El auto-diagnóstico se sustenta en un conjunto de competencias investigativas hasta el nivel de transferencia de habilidades. Además de contribuir a la asignación idónea del capital humano en proyectos, el sistema permite establecer un plan estratégico de formación por lo que fue implementado mediante el desarrollo de dos aplicaciones: de escritorio y Web conectadas a una base de datos para lograr la obtención de resultados en tiempo real.

\section{FUNDAMENTACIÓN TEÓRICA}

El desarrollo del sistema se enmarca en el contexto de la Sociedad del conocimiento, en los indicadores científicos estandarizados internacionalmente para medir la producción científica y tecnológica, y en las competencias de investigación necesarias para la generación de conocimiento y su recapitalización en las empresas (Romo y Villalobos, 2016). En la Figura 1 se muestran dos de los ocho pasos que conforman el modelo donde se inserta el sistema:

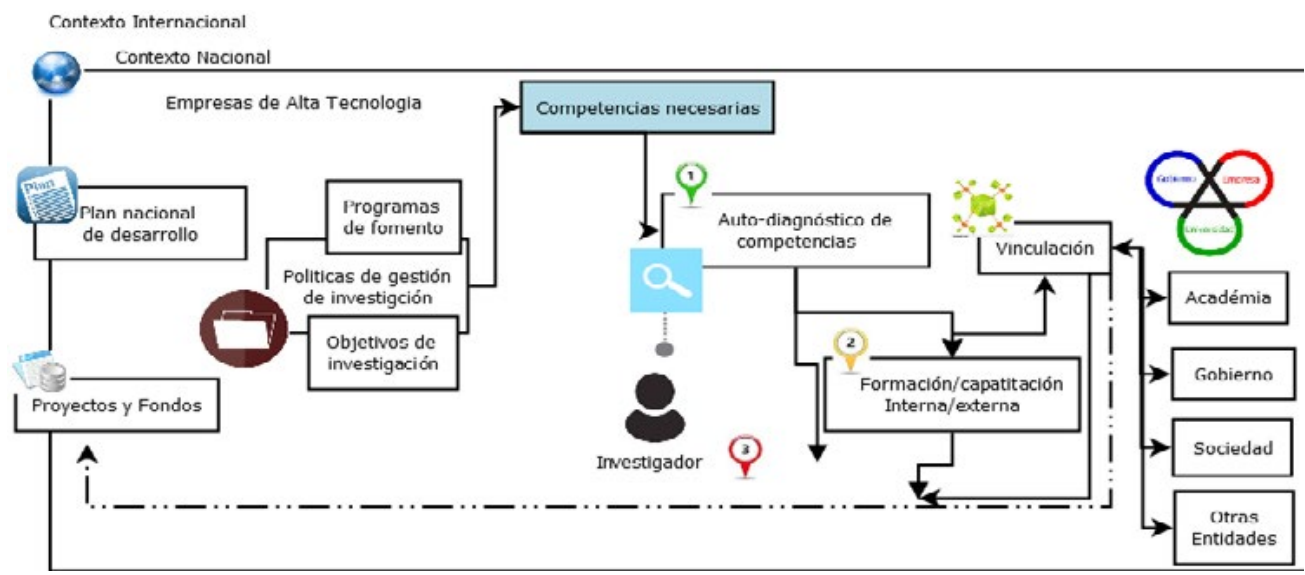

Fig. 1. Pasos 1 y 2 del modelo de gestión del conocimiento para el desarrollo de competencias de investigación en empresas de alta tecnología. Fuente: Romo (2015).

Un Auto-diagnóstico de competencias les permite a los individuos "identificar y reconocer su propia potencia y habilidad para enfrentarse al mundo de la organización" (García, Olvera, Elizondo y López, 2013). En el modelo, el 
Auto-diagnóstico contribuye a establecer el nivel de habilidades del capital humano con instrumentos de medición y diagnóstico recapitalizando el conocimiento (Cameron y Whetten, 2013).

Caravaca, González, García, Fernández y Mendoza (2014) señalan que el contexto económico competitivo en él que se desempeñan las empresas tiene como principal activo el conocimiento por la re-organización de la sociedad económica y políticamente alrededor de la información y la explotación del conocimiento como variables de la distribución del poder (Tedesco, 1999) sustentándose cada vez menos en la transformación de materias primas (Aróstica, 2014; Barroso, 2013; Castells, 2011; Leigh, y Blakely, 2013).

En el contexto de la sociedad del conocimiento, la educación y el aprendizaje son esenciales para el desarrollo de competencias que garanticen la equidad social (Aretio, 2012; Tobón, 2012; Organización de las Naciones Unidas para la Educación, la Ciencia y la Cultura (UNESCO, 2005, 2010; Zapata-Ros, 2012) por la exigencia de capital humano especializado requerido en las empresas.

El Banco Mundial (BM, 2012) y la Comisión Económica para América Latina (CEPAL, 2000) indican, que la transición hacia la sociedad del conocimiento depende de la postura de los gobiernos con respecto a sus políticas macroeconómicas para la producción del conocimiento.

Los países de América Latina han aceptado la necesidad de orientar sus esfuerzos hacia la explotación del conocimiento científico a través de políticas que garanticen una economía competitiva generadora de empleos (Ocampo, Camarena, y Luna, 2011).

Dado que el principal activo de las empresas es un bien intangible que genera valor en las industrias, Vilaseca, Torrent, y Lladós (2013) establecen que el "conocimiento será económicamente relevante siempre y cuando se manifieste en la actividad económica", su incorporación se basa en los criterios de importancia y necesidad por lo que Akehurst, Rueda, Armengot, López, y Marqués (2011) identifican los tipos de conocimiento en las organizaciones volviendo imprescindible las competencias.

\section{Competencias}

La necesidad de identificar las competencias para maximizar el desempeño laboral se hizo evidente en países como Alemania (Dalichow, 1997; Miklitsch, 1997), el Reino Unido, la Unión Europea (Agencia para la Calidad de la Educación Superior [QAA], 2008), Estados Unidos (Tucker y Ruzzi, 2000), Canadá (Kobinger, 2000) y México (Argüelles, 1996; Ibarra, 1996, 1998). El estudio de McClelland (1996) demostró que no existe correlación entre coeficiente intelectual y éxito, sino que también se encuentran asociados factores como: atributos personales, aptitudes y motivaciones.

Como resultado de la coexistencia de diversos paradigmas (Hernández-Rojas, 2000), el concepto de competencia puede abordarse desde diversas perspectivas, desde de pensamiento complejo (Restrepo, Sarmiento y Ramos, 2003) se concibe como: 
(...) procesos complejos de desempeño con idoneidad en determinados contextos, integrando diferentes saberes (saber ser, saber hacer, saber conocer y saber convivir), para realizar actividades y/o resolver problemas con sentido de reto, motivación, flexibilidad, creatividad, comprensión y emprendimiento, dentro de una perspectiva de procesamiento metacognitivo, mejoramiento continuo y compromiso ético, con la meta de contribuir al desarrollo personal, la construcción y afianzamiento del tejido social, la búsqueda continua del desarrollo económico-empresarial sostenible, y el cuidado y protección del ambiente y de las especies vivas (Tobón, 2008, p.3).

Con base en las teorías desde donde se definen, la taxonomía de las competencias está sujeta a diversas interpretaciones (Fernández -Salinero, 2006), pero hay consenso en la clasificación general en tres tipos: laboral, profesional y educativa (Charria et al., 2011).

\section{Competencias en investigación}

Los ejes cognitivos abordados en las competencias del que se deriva su evaluación dependen en gran medida de su tipificación. Relacionados con estas competencias Rivera Heredia (2009) establece seis dominios de evaluación sintetizados en la tabla 1.

Tabla 1. Relación de competencias básicas y genéricas con el dominio para habilidades de investigación.

\begin{tabular}{|c|c|c|c|}
\hline Tipo & Dominio & Competencias & Desglose \\
\hline $\begin{array}{l}\text { Básica, } \\
\text { Genérica o trans- } \\
\text { versal }\end{array}$ & $\begin{array}{l}\text { Metodológico. } \\
\text { (Nuñez, 2007; Muñoz, } \\
\text { Quintero y Munévar, } \\
\text { 2001) }\end{array}$ & $\begin{array}{l}\text { Capacidad para reali- } \\
\text { zar las fases del proce- } \\
\text { so de investigación }\end{array}$ & $\begin{array}{l}\text { Interpretativa, } \\
\text { Argumentativa, } \\
\text { Propositiva, sisté- } \\
\text { mica, Resolución/ } \\
\text { problemas }\end{array}$ \\
\hline Básica & $\begin{array}{l}\text { Tecnológico. } \\
\text { (Izaguirre, Baká y Obre- } \\
\text { gón, 2009) }\end{array}$ & $\begin{array}{l}\text { Capacidad para } \\
\text { emplear herramientas } \\
\text { tecnológicas }\end{array}$ & $\begin{array}{l}\text { Gestión de la Infor- } \\
\text { mación y competen- } \\
\text { cia digital }\end{array}$ \\
\hline $\begin{array}{l}\text { Genérica o trans- } \\
\text { versal }\end{array}$ & $\begin{array}{l}\text { Búsqueda de informa- } \\
\text { ción. } \\
\text { (Molina-Ordóñez, Hua- } \\
\text { maní y Mayta-Tristán, } \\
\text { 2008; Correa, 2009) }\end{array}$ & $\begin{array}{l}\text { Capacidad para obten- } \\
\text { ción de información en } \\
\text { diversos medios }\end{array}$ & $\begin{array}{l}\text { Gestión de recursos } \\
\text { Gestión de informa- } \\
\text { ción }\end{array}$ \\
\hline $\begin{array}{l}\text { Genérica o trans- } \\
\text { versal }\end{array}$ & $\begin{array}{l}\text { Trabajo en grupos de } \\
\text { investigación. } \\
\text { (Fandos, 2006; Maldona- } \\
\text { do et al., 2007; Barraza, } \\
\text { 2007) }\end{array}$ & $\begin{array}{l}\text { Capacidad para la } \\
\text { gestión y el trabajo } \\
\text { colaborativo }\end{array}$ & $\begin{array}{l}\text { Trabajo en equipo } \\
\text { Planificación del } \\
\text { trabajo }\end{array}$ \\
\hline Básica & $\begin{array}{l}\text { Exposición de resulta- } \\
\text { dos. } \\
\text { (Nuñez, 2007; } \\
\text { Correa, 2009) }\end{array}$ & $\begin{array}{l}\text { Capacidad de argu- } \\
\text { mentación y exposición } \\
\text { de ideas }\end{array}$ & $\begin{array}{l}\text { Comunicación oral } \\
\text { y escrita (COyE) en } \\
\text { la lengua materna/ } \\
\text { extranjera }\end{array}$ \\
\hline Básica & $\begin{array}{l}\text { Difusión, divulgación y } \\
\text { diseminación de resul- } \\
\text { tados } \\
\text { (Muñoz, et. al, 2001) }\end{array}$ & $\begin{array}{l}\text { Capacidad de redac- } \\
\text { ción y síntesis }\end{array}$ & $\begin{array}{l}\text { Comunicación oral y } \\
\text { escrita en la lengua } \\
\text { materna/extranjera }\end{array}$ \\
\hline
\end{tabular}


En la categorización de las competencias investigativas sobresalen el dominio metodológico en el proceso de gestión de proyectos de forma colaborativa sustentada en el uso de la tecnología para la realización efectiva de búsqueda y análisis de información, así como las competencias para la argumentación oral y la comunicación escrita de resultados, por lo que su eficiente gestión deriva en una mayor comprensión de los procesos científicos que permiten incrementar los desarrollos tecnológicos.

\section{MATERIALES Y MÉTODOS}

El método empleado fue el de Desarrollo tecnológico para el "Desarrollo de proyectos" del área de ingeniería y tecnología en la disciplina de Ingeniería de software. Dentro del modelo de gestión del conocimiento para el desarrollo de competencias investigativas en empresas de Alta Tecnología, el sistema del auto-diagnóstico se inserta en la fase 1 de acuerdo con la figura 2:

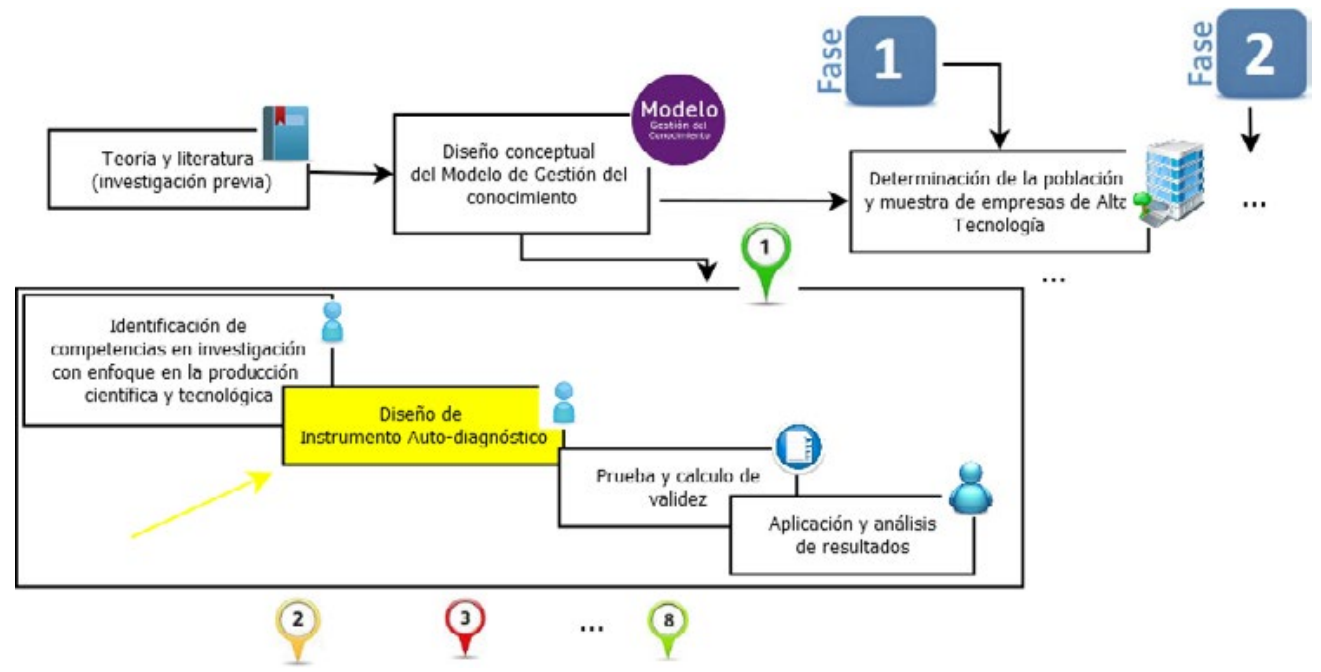

Figura 2. Fase 1, etapa 1 del diagrama del método para el sistema de Auto-diagnóstico.

El desarrollo del sistema se compone de cuatro etapas para las dos aplicaciones que lo constituyen, la de escritorio y el sitio web:

-Análisis y diseño del sistema integral.

-Desarrollo e implementación de aplicaciones.

-Integración del sistema.

-Pruebas y correcciones.

En la etapa de análisis y diseño se genera un documento de requerimientos estandarizado en el que se encuentran los diagramas de identificación de actores, casos de uso, identificación de entidades, diagrama de clases, diagrama de secuencia, colaboración y estado, así como el diagrama entidad-re- 
lación para la base de datos del servidor central y el diseño de imágenes e iconos de las aplicaciones.

Para el levantamiento de requerimientos se utilizó la técnica del cuestionario personalizado, el cuestionario se realiza mediante entrevista al capital humano que pertenece al área de investigación, consta de 25 preguntas 15 abiertas 10 cerradas, con ellas se determinan los requerimientos funcionales, no funcionales y de sistema. Para la etapa de desarrollo e implementación de aplicaciones se requiere:

- Diseñar e implementar una Base de datos para el registro del capital humano del área de investigación y los resultados de los auto-diag nósticos.

- Implementar el sistema de la aplicación central considerada como "de escritorio" capaz de mantener el control de los expedientes del capital humano en el área de investigación y desarrollo.

- Crear un sitio web para realizar el auto-diagnóstico en línea.

- Administrar sesiones para los diferentes tipos de usuarios y garanti zar el acceso y la seguridad del sistema.

Durante la etapa de integración del sistema se establecen las conexiones de las aplicaciones con la base de datos y se desarrollan los mecanismos para garantizar la seguridad sobre los accesos a los datos.

En la etapa de Pruebas y correcciones se asiste a la empresa con el objetivo de que colaboren en las pruebas los distintos usuarios de Sistema. Se corrigen los posibles errores y se mantiene en uso la aplicación durante el periodo de un mes para garantizar y prevenir problemas futuros en el acceso a registro o corrupción en los datos.

A partir del diseño del diagrama Entidad-Relación se desarrolla la base de datos (BD) central que contiene las instrucciones para construir la BD, las tablas y los registros. Su flexibilidad se encuentra en el tipo de conexión establecida, lo que hace posible migrar los datos a cualquier gestor a la que se conectan las dos aplicaciones del sistema.

\section{RESULTADOS}

Se presentan los resultados enfocados en el desarrollo e implementación del sistema que permitió controlar el proceso de registro del capital humano de las empresas de alta tecnología, actualizar su información, así como crear un registro de las competencias en el área de investigación El esquema general para la base de datos del sistema para el auto-diagnóstico se muestra en la Figura 3 y en la figura 4 un ejemplo de la evaluación por competencias con su composición por factores. 


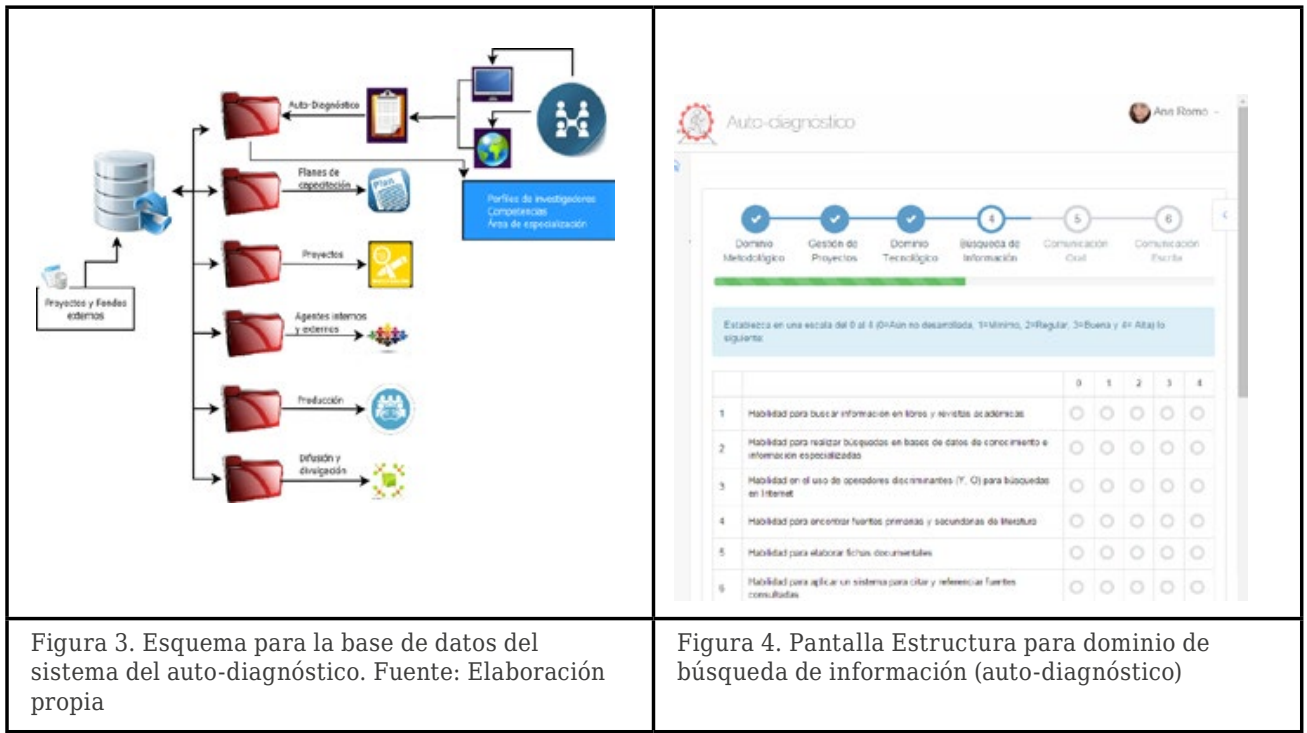

La funcionalidad del sistema se basa en menús de opciones al usuario para el acceso a los dominios de evaluación del Auto-diagnóstico de competencias investigativas mediante una escala de Likert. La escala tiene los siguientes criterios:

$0=$ Aún no desarrollada.

1=Mínimo.

$2=$ Regular.

$3=$ Buena.

$4=$ Alta.

Para establecer la validez del instrumento se realizó validez de contenido, criterio y constructo del Auto-diagnóstico.

Los dos componentes principales del sistema: escritorio y web se integran y se conectan a la base de datos en el servidor central con el objetivo de lograr la conexión de forma remota desde las aplicaciones que operan dos entornos y que los usuarios puedan realizar el auto-diagnóstico desde cualquier navegador. Una vez que se ingresa al sistema, se pueden evaluar seis dominios de competencias.

Para los resultados del sustentante, se diseñó un Informe en el que se especifica el código de evaluación e información relativa al nivel de competencias para cada uno de los dominios definidos con rangos e interpretación, nivel de habilidades y una breve descripción del conjunto de "debilidades" y "fortalezas" que posee.

Los resultados del sistema facilitan el análisis de requerimientos del capital humano dedicado a labores de investigación en las empresas de Alta Tecnología así como la definición de planes de capacitación para el personal y el establecimiento de proyectos de acto impacto que fortalezcan la producción científica y tecnológica del país. 


\section{CONCLUSIONES}

El análisis de resultados del auto-diagnóstico permite identificar las fortalezas y debilidades de los participantes en los diferentes dominios de competencias por lo que su aplicación en viable en las empresas.

La obtención de resultados facilita la definición del perfil del capital humano que se desempeña en la industria partiendo de la identificación de las fortaleces y debilidades establecidas por los propios participantes, con lo que se establecen planes generales y personalizados de capacitación para la generación de portafolios de proyectos y protocolos que contribuyan a la obtención de financiamiento a través de participación en convocatorias de fondos estatales fortaleciendo el capital estructural de las industrias.

La aplicación del auto-diagnóstico posibilita la obtención de resultados en línea a través de los accesos de usuarios por lo que es indispensable la seguridad de los sistemas y su sincronización en tiempo real para garantizar la consistencia de los datos.

Entre las principales ventajas del sistema de auto-diagnóstico se encuentra el establecimiento del nivel de desarrollo de competencias en el área de investigación en seis dominios presentando resultados confiables de manera eficiente y rápida.

\section{REFERENCIAS BIBLIOGRÁFICAS}

Agencia para la calidad de la Educación Superior. (2008). The framework for higher education qualifications in England, Wales and Northern Ireland. QAA. Quality Assurance Agency for Higher Education. http://www.qaa. ac.uk/en/Publications/Documents/Framework-Higher-Education-Qualifications-08.pdf

Akehurst, G., Rueda, C., López, S. V. y Marqués, D. P. (2011). Ontological supports of knowledge: knowledge creation and analytical knowledge. Management Decision, 49(2), 183-194.

Aretio, L. G. (2012). Sociedad del conocimiento y educación. Universidad Nacional de Educación a Distancia. Madrid. Libro electrónico. Recuperado de: http://e-spacio.uned.es/fez/eserv.php?pid=bibliuned:UNESCO-libros-sociedad_conocimiento\&dsID=Documento.pdf

Argüelles, A. (1996). Competencia laboral y educación basada en normas de competencia. Noriega Editores. México.

Aróstica, P. (2014). China en transición a la sociedad del conocimiento: implicaciones para América Latina. Documento de proyecto. Comisión Económica para América Latina y el Caribe (CEPAL). Naciones Unidas. Santiago de Chile.

Barraza, A. (2007). La formación docente bajo una conceptualización comprehensiva y un enfoque por competencias. Estudios pedagógicos, 33(2), 131-153.

Barroso, C. (2013). La sociedad del conocimiento y el entorno digital. Teoría de la educación, educación y cultura en la sociedad de la información, 14(3), 61-86.

Banco Mundial. (2012). Estadísticas de los países. Gasto en investigación y 
desarrollo \% del PIB. Recuperado de: http://datos.bancomundial.org/indicador/GB.XPD.RSDV.GD.ZS

Cameron, K. S. y Whetten, D. A. (2013). Organizational effectiveness: A comparison of multiple models. Academic Press.

Caravaca, I., González, G., García, A., Fernández, V. y Mendoza, A. (2014). Conocimiento, innovación y estrategias públicas de desarrollo: análisis comparado de tres ciudades medias de Andalucía (España). EURE, 40(119), 49-74.

Castells, M. (2011). The Rise of the Network Society: The information age: Economy, society, and culture. John Wiley \& Sons.

Charria Ortiz, V. H., Sarsosa Prowesk, K. V., Uribe Rodríguez, A. F., López Lesmes, C. N. y Arenas Ortiz, F. (2011). Definición y clasificación teórica de las competencias académicas, profesionales y laborales: Las competencias del psicólogo en Colombia. Psicol. Caribe, (28), 133-165.

Comisión Económica para América Latina y El Caribe. (2000). América Latina y El Caribe en la transición hacia una sociedad del conocimiento. Una agenda de políticas públicas. CEPAL. Recuperado de: http://www.cepal. org/publicaciones/xml/2/4312/lcl1383e.pdf

Correa, B. (2009). Medición de las competencias investigativas en docentes de fisiología: una aproximación empírica. Rev. Fac. Med. (Bogotá), 57(3), 205 217.

Dalichow, F. (1997). A Comparison of Credit Systems in an International Context. Ministerio de Educación, Ciencia, Investigación y Tecnología de la República Federal Alemana, Bonn.

Fandos, M. (2006). El cambio educativo: nuevos escenarios y modalidades de formación. Revista Educar 38, 243-258.

Fernández-Salinero, C. (2006). Las competencias en el marco de la convergencia europea: Un nuevo concepto para el diseño de programas educativos. Encounters on Education, 7.

García, G., Olvera A., Elizondo, M. y López, S. (2013). Tres competencias esenciales de los profesionales inteligentes: advertir y responder, combinar y conectar, y crear y producir. Sotavento MBA, (21), 90-107.

Hernández-Rojas, G. (2000). Paradigmas en psicología de la educación. Editorial Paidós.

Ibarra, A. (1996). El sistema normalizado de competencia laboral. Competencia laboral y educación basada en normas de competencia. Noriega Editores. México.

. (1998). El desarrollo de los sistemas normalizado y de certificación de competencia laboral y transformación de la formación y capacitación. México: CONOCER, 1998. Presentado al Seminario Andino sobre Formación Basada en Competencia Laboral, Bogotá, 1998. La formación de los administradores en la sociedad del conocimiento: retos y oportunidades. Gestión y Estrategia, 15(2), 3.

Izaguirre, R., Baká, P. y Obregón, C. (2009). Las competencias para el trabajo científico: una necesidad de la formación profesional. Universidad Nacional de Guinea Ecuatorial, Facultad de Ciencias Médicas.

\section{Cumbres}


Kobinger, N. (2000). El sistema de formación profesional y técnica por competencias desarrollado en Quebec. Competencia laboral y educación basada en normas de competencia.

Leigh, N. \& Blakely, E. (2013). Planning local economic development: Theory and practice. SAGE Publications, Incorporated.

Maldonado, L., Landazábal, D., Hernández, J., Ruíz, Y., Claro, A., Vanegas, H. y Cruz, S. (2007). Visibilidad y formación en investigación. Estrategias para el desarrollo de competencias investigativas. Revista Studiositas, 2(2), 43-56.

McClelland, D. C. (1996). Does the field of personality have a future? Journal of Research in Personality, 30(3), 429-434.

Miklitsch, R. (1997). The Risks and Rewards of (Anti-) Transference. Education and Cultural Studies: Toward a Performative Practice, 259.

Mínguez, R. y Hernández, M. A. (2013). Hacia otra educación en la sociedad del conocimiento: cuestiones y propuestas pedagógicas. Teoría de la Educación. Educación y Cultura en la Sociedad de la Información, 14(3). Recuperado de: http://dialnet.unirioja.es/servlet/articulo?codigo $=4509641$

Molina-Ordóñez, J., Huamaní, C. y Mayta-Tristán, P. (2008). Apreciación estudiantil sobre la capacitación universitaria en investigación: estudio preliminar. Rev Peru Med Exp Salud Publica, 25(3), 325-29.

Moreno-Bayardo, M. (2005). Potenciar la educación. Un currículum transversal de formación para la investigación. REICE-Revista Electrónica Iberoamericana sobre Calidad, Eficacia y Cambio en Educación, 3(1).

Muñoz, J., Quintero, J. y Munévar, R. (2001). Competencias investigativas para profesionales que forman y enseñan: ¿cómo desarrollarlas? Coop. Editorial Magisterio.

Núñez, N. (2007). Desarrollo de Habilidades para la Investigación (DHIN). Revista Iberoamericana de Educación, 44(6), 7.

Ocampo, B. F., Camarena G. P. y Luna C. R. de. (2011). Los desafíos de las instituciones de educación superior de México en la sociedad del conocimiento. Innovación Educativa, 57 (11), 207-212.

Organización de las Naciones Unidas para la Educación, la Ciencia y la Cultura. (2005). Towards Knowledge Societies. World Report. UNESCO Publishing: París.

. (2010). Informe de la UNESCO sobre la Ciencia 2010. El estado actual de la ciencia en el mundo. Ediciones Unesco: París.

Organización para la Cooperación y el Desarrollo Económicos. (1997). Exámenes de las políticas nacionales de la educación. México-Educación Superior. Francia: OCDE.

Plan Nacional de Desarrollo 2013-2018 (2013). Gobierno de la República de México. Recuperado de: http://pnd.gob.mx/.

Restrepo, G., Sarmineto, J. y Ramos, J. (2003). Competencias y pedagogías en la enseñanza de las ciencias sociales. Trazas y Miradas: Evaluación y competencias. Universidad Nacional de Colombia. Unibiblos. Bogotá.

Rivera Heredia, M. (2009). La evaluación de las habilidades de investigación de los estudiantes de Educación Superior: propuesta de un instrumento. 
Recuperado de: http://www.comie.org.mx/congreso/memoriaelectronica/ v09/ponencias/at01/PRE1178867349.pdf

Romo, A. y Villalobos Alonzo, M. (2016). Knowledge Management Model for Scientific and Technological Production. IOSR Journal of Business and Management (IOSR-JBM), 10(III), 114-124.

Romo, A. (2015). Modelo de gestión del conocimiento para el desarrollo de competencias de investigación en empresas de Alta Tecnología en México. Tesis Doctoral. Planeación estratégica y dirección tecnológica. Universidad Popular Autónoma del Estado de Puebla (UPAEP). México.

Romo, A., Villalobos. A. y Toríz, A. (2015) Estado de la Ciencia y Tecnología en México a partir de los principales indicadores sintéticos. Academia Journals, 1(13), 2086-2091.

Secretaría de Economía. (2013). Programa de desarrollo innovador 20132018. Secretaria de Economía. Gobierno de la república de México. Recuperado de: http://www.economia.gob.mx/conoce-la-se/programas-se/ programa-de-desarrollo-innovador-2013-2018

Tedesco, J. (1999). La educación y la sociedad del conocimiento y de la información. Encuentro Internacional de Educación Media-IIPE. Recuperado de: www.pedagogica.edu.co/storage/rce/articulos/rce36-37_09controv.pdf

Tobón, S. (2012). El aprendizaje por proyectos formativos: hacia la transformación real de las prácticas docentes. 1er. encuentro internacional de educación, los desafíos de la educación: una visión hacia el 2020. Recuperado de: http://www.youtube.com/watch?v=fZPNJsFBcFU

. (2008). La formación basada en competencias en la educación superior: el enfoque complejo. México: Universidad Autónoma de Guadalajara.

Tucker, M. \& Ruzzi, B. B. (2000). Un sistema nacional de normas de competencia y certificaciones para los Estados Unidos: etapas iniciales de implementación. Boletín Cinterfor: Boletín Técnico Interamericano de Formación Profesional, 149, 201-216.

Vilaseca, J., Torrent, J. y Lladós, J. (2013). De la economía de la información a la economía del conocimiento: algunas consideraciones conceptuales y distintivas. Revista Tendencias, 2(2).

Zapata-Ros, M. (2012). Teorías y modelos sobre el aprendizaje en entornos conectados y ubicuos. Bases para un nuevo modelo teórico a partir de una visión crítica del conectivismo. Recuperado de: http://eprints.rclis.org/17463/1/bases_teoricas.pdf

\section{Cumbres}

